\title{
Effects of red beetroot supplementation on gut microbiome and short chain fatty acid profiles in weaned pigs
}

\author{
O. O. Adekolurejo ${ }^{1,2}$, K. McDermott ${ }^{2}$, H. M. Miller ${ }^{2}$, H.M.R. Greathead ${ }^{2}$, A. R. Mackie ${ }^{1}$ and \\ C. Boesch ${ }^{1}$ \\ ${ }^{1}$ School of Food Science and Nutrition and \\ ${ }^{2}$ School of Biological Sciences University of Leeds, Leeds, UK
}

Weaned pigs are prone to gut dysbiosis and microbiota disruptions that result in diarrhoea and impaired growth. Dietary modulation of the gut bacterial composition and metabolism plays a vital role in proliferation of beneficial bacteria to alleviate this condition ${ }^{(1)}$. Currently, weaned pig diet is supplemented with pharmacological levels of zinc oxide, however, due to increased emergence of antibiotic resistance and environmental pollution, there is pressure for replacements ${ }^{(2)}$. Red beetroot consumption has been associated with numerous health benefits ${ }^{(3)}$ but little is known about its effects on the gut microbiota. Therefore, in this study, we assessed the effects of supplemental red beetroot on the gut microbiota and faecal short chain fatty acid (SCFA) production in weaned pigs.

Intestinal digesta samples from jejunum, ileum and caecum were collected from 38 weaning pigs fed one of four diet which comprised; a control diet (CON) or CON diet supplemented with zinc oxide $(3000 \mathrm{mg} / \mathrm{kg}, \mathrm{ZNO})$ or red beetroot $(2 \%-20 \mathrm{~g} / \mathrm{kg}, \mathrm{RB} 2 ; 4 \%$ $40 \mathrm{~g} / \mathrm{kg}$, RB4) for 14 days. The gut microbiome was characterised by $16 \mathrm{~s}$ rRNA sequencing of the V4 region in DNA isolated from the intestinal digesta samples. Sequence reads were processed with Mothur, and results analysed in R (v 4.0.0) and Graph pad Prism 9. Faecal short chain fatty acid profiles were assayed using gas chromatography.

Whilst no differences were observed in the population of Lactococcus, Lactobacillus and Lactobacillales in the gut of the pigs, there was a significant increase in the genera Enterobacteriaceae, Pasturellaceae and Campylobacter in the caecum of pigs on RB4 diet. Nevertheless, RB2 diet increased the bacterial diversity of the gut (Chao 1 index; $\mathrm{P}<0.05$ ); with 47 and 21 genera more than pigs fed CON and ZNO diets, respectively. Also, RB2 $(\mathrm{R} 2=0.080, \mathrm{P}=0.006)$ and $\mathrm{ZNO}(\mathrm{R} 2=0.063, \mathrm{P}=0.042)$ diets significantly increased the bacterial composition of the caecum $(\mathrm{R} 2=0.177, \mathrm{P}=0.013$ ), while no difference was observed in the bacterial composition of the jejunum and ileum $(\mathrm{R} 2=0.041, \mathrm{P}=0.051)$. The diets were highly similar for the faecal SCFA levels, however faecal acetate concentration was significantly lower in pigs on $\mathrm{ZNO}(\mathrm{P}=0.0005)$ and $\mathrm{RB} 4(\mathrm{P}=0.02)$ diets compared to the control.

In summary, weaned pig diet supplemented with red beetroot has the potential to increase the microbial diversity, modulate proliferation of beneficial bacteria and lower faecal acetate concentration compared to CON and ZNO diets. However, care should be taken at higher inclusion levels to avoid the increase of potential pathogenic bacteria species in the gut. The importance of these outcomes for humans needs to be established.

\section{Acknowledgements}

We appreciate the funds provided by the Commonwealth Scholarship Commission.

\section{References}

1. Celi P, Cowieson AJ, Fru-Nji F, et al. (2017) Ani Feed Sci Tech 234, 88-100.

2. Ciesinski L, Guenther S, Pieper R, et al. (2018) Plos One 13, E0191660.

3. Rahimi P, Abedimanesh S, Mesbah-Namin SA et al. (2018) Crit Rev Food Sci Nutr, 1-30. 